\title{
Investigating Engineering Students' Understandings of Social and Ethical Responsibility: Coding Framework and Initial Findings
}

\author{
Prof. Brent K. Jesiek, Purdue University, West Lafayette (College of Engineering)
}

Dr. Brent K. Jesiek is an Associate Professor in the Schools of Engineering Education and Electrical and Computer Engineering at Purdue University. He also leads the Global Engineering Education Collaboratory (GEEC) research group, and is the recipient of an NSF CAREER award to study boundary-spanning roles and competencies among early career engineers. He holds a B.S. in Electrical Engineering from Michigan Tech and M.S. and Ph.D. degrees in Science and Technology Studies (STS) from Virginia Tech. Dr. Jesiek draws on expertise from engineering, computing, and the social sciences to advance understanding of geographic, disciplinary, and historical variations in engineering education and practice.

\section{Dr. Carla B. Zoltowski, Purdue University, West Lafayette (College of Engineering)}

Carla B. Zoltowski is an assistant professor of engineering practice in the Schools of Electrical and Computer Engineering and (by courtesy) Engineering Education at Purdue University. She holds a B.S.E.E., M.S.E.E., and Ph.D. in Engineering Education, all from Purdue. Prior to this she was Co-Director of the EPICS Program at Purdue where she was responsible for developing curriculum and assessment tools and overseeing the research efforts within EPICS. Her academic and research interests include the professional formation of engineers, diversity and inclusion in engineering, human-centered design, engineering ethics, leadership, service-learning, and accessibility and assistive-technology.

\section{Debra S. Fuentes, Brigham Young University}

Debra S. Fuentes is a doctoral student at Brigham Young University in Educational Inquiry, Measurement, and Evaluation specializing in Mathematics Education. She received a Master's degree in Curriculum and Instruction emphasizing English as a Second Language, and a Bachelor's degree in elementary education, minoring in Spanish and pre-medicine studies. She previously worked in education as a teacher and administrator in Utah and Mexico for eleven years combined. Much of her current work and research focuses on Cognitively Guided Instruction in mathematics.

\section{Dr. Stephanie Claussen, Colorado School of Mines}

Stephanie Claussen's experience spans both engineering and education research. She obtained her B.S. in Electrical Engineering from the Massachusetts Institute of Technology in 2005. Her Ph.D. work at Stanford University focused on optoelectronics, and she continues that work in her position at the Colorado School of Mines, primarily with the involvement of undergraduate researchers. In her role as an Associate Teaching Professor, she is primarily tasked with the education of undergraduate engineers. In her courses, she employs active learning techniques and project-based learning. Her previous education research, also at Stanford, focused on the role of cultural capital in science education. Her current interests include engineering students' development of social responsibility and the impact of students' backgrounds in their formation as engineers.

\section{Dr. Gregg Morris Warnick, Brigham Young University}

Gregg M. Warnick is the Director of the Weidman Center for Global Leadership and Associate Teaching Professor of Engineering Leadership within the Ira A. Fulton College of Engineering and Technology at Brigham Young University (BYU). The center provides oversight for leadership development and international activities within the college and he works actively with students, faculty and staff to promote and develop increased capabilities in global agility and leadership. His research and teaching interests include developing global agility, globalization, leadership, project management, ethics, and manufacturing processes. Gregg has lived in numerous locations within the USA and Europe and has worked in many places including North America, South America, Europe, Asia, and Africa. Prior to joining BYU, Gregg worked for Becton Dickinson, a Global Medical Technology fortune 500 Company. In this capacity he 
worked as a product development engineer, quality engineer, technical lead, business leader and program/project manager managing many different global projects. Gregg received his $\mathrm{PhD}$ in Educational Leadership and Higher Education from the University of Nebraska-Lincoln with a Master of Technology Management degree and a BS in Manufacturing Engineering Technology, from Brigham Young University. Gregg also does consulting in project management and leadership working with IPS Learning and Stanford University where he provides training for fortune 500 companies throughout the world. 


\section{Investigating Engineering Students' Understandings of Social and Ethical Responsibility: Coding Framework and Initial Findings}

\section{Introduction}

Examples of unethical and irresponsible practices by practicing engineers continue to make headlines. The National Academy of Engineering and other organizations have issued numerous calls to cultivate greater social and ethical responsibility among engineering students and professionals. These drivers have motivated the creation of many formal ethics courses and programs across engineering education, as well as other interventions (e.g., service learning programs) to more broadly challenge engineering students to see themselves as engaged citizens and community members. However, there is a lack of research on foundational understandings of social and ethical responsibility among undergraduate engineering students, both in terms of what these specific constructs mean to students, as well as how their views change over time and are impacted by specific kinds of learning experiences.

In an effort to investigate how students perceive social and ethical responsibility, and especially how these perceptions change over the course of a four-year engineering degree program, this study explores the following objectives:

O1) Characterize patterns of ethical development among undergraduate engineering students,

O2) Identify specific context variables (e.g., climate and culture of programs and institutions) and types of interventions (e.g., formal ethics instruction, service learning programs, etc.) that have positive (or negative) impacts on foundational measures and understandings of social and ethical responsibility, and

O3) Identify specific student characteristics that can be leveraged to grow programs oriented toward social and/or ethical responsibility, while increasing program alignment with and impacts on - participating individuals.

In order to accomplish these objectives, this study involves a longitudinal mixed-methods design collecting and analyzing quantitative and qualitative data from undergraduate engineering students at four universities over a four-year period as shown in Figure 1. The purpose of this study is twofold: (a) to expand the current research on engineering, ethics, and social responsibility, and (b) improve implementation of highly effective institutional interventions that develop socially and ethically responsible engineering leaders and change agents.

The current study supports the aforementioned project objectives by investigating two main research questions:

RQ1) What do engineering students perceive as responsible (and irresponsible) professional conduct, and what do they perceive as just (and unjust) professional work practices? and 
RQ2) How do foundational measures and understandings of social and ethical responsibility change during a four-year engineering degree program, both in general and in relation to specific learning environments and experiences?

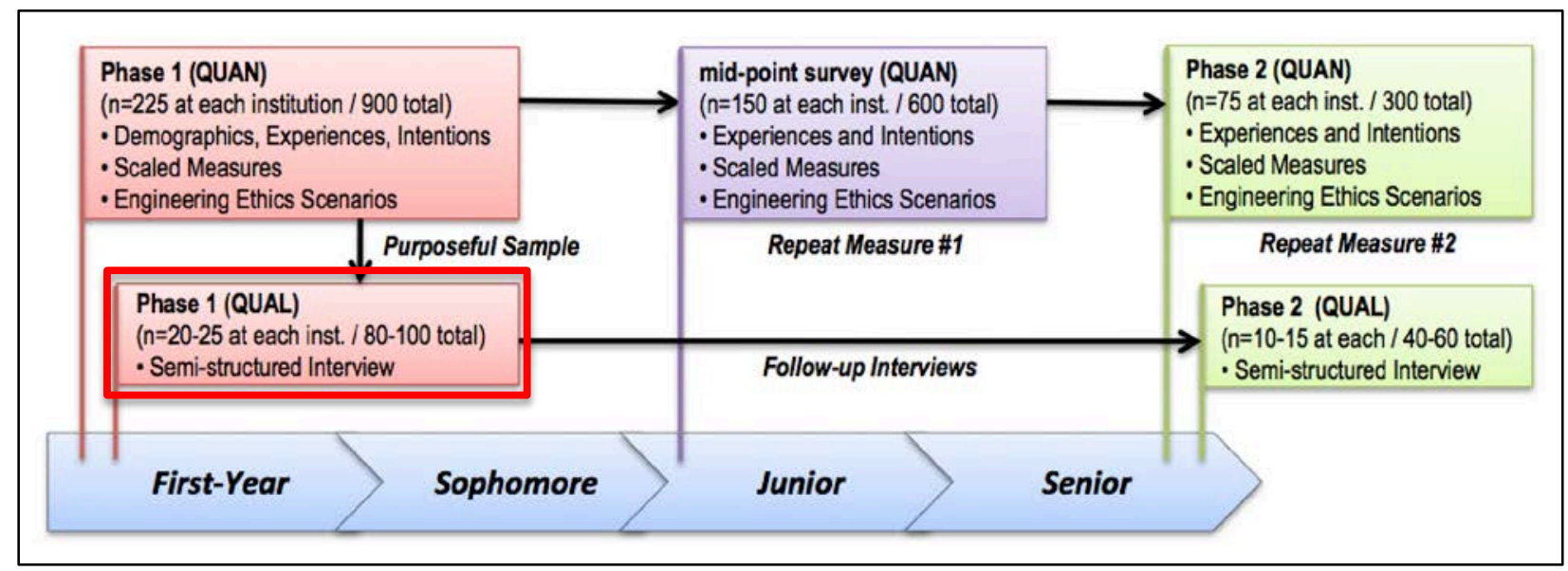

Figure 1. Study Design Overview - Phase 1 (QUAL) is the Focus of this Paper

The study includes collecting data at three different points in the students' undergraduate experience:

- Phase 1: Baseline survey and interviews during the first semester/year

- Mid-point: Mid-point survey during the junior year ( $5^{\text {th }}$ semester), and

- Phase 2: Survey and interviews during their senior year ( $8^{\text {th }}$ semester). The survey includes items and measures related to engineering ethics knowledge, justice beliefs, political and social involvement, macro-ethical considerations, moral attentiveness, moral disengagement, and ethical climate, along with extensive demographic measures. The interview protocol explored these areas through questions related to: 1) general definitions of ethics and engineering ethics, including macro-ethics, 2) experiences (past, present, and future) that may shape students' ethical perspectives and sense of social responsibility, 3) ethical climate, and 4) ethical scenarios.

The baseline data collection included student survey responses from four collaborating universities within the United States representing different geographic locales and university types. The collaborating universities included a public research-intensive and project-based university in the Southwest (Arizona State University); a private, religious, and researchintensive university located in the mountain West (Brigham Young University); a public, primarily undergraduate-serving university located in the mountain West (Colorado School of Mines); and a public research-intensive university located in the Midwest (Purdue University). Preliminary analysis of the survey data has been completed and results published in the proceedings of the 2016 ASEE Annual Conference and Exposition. ${ }^{1}$

This paper reports on the qualitative portion of the first phase of data collection. It includes development of a qualitative research protocol, informed by the survey data and resulting in a codebook used to analyze interview data from an initial 29 of 113 follow-up interviews to a survey administered to over 780 first-semester engineering students. 
The coding framework is organized into three major areas:

- perspectives in specific areas of interest such as general definitions of ethics and engineering ethics, views on the university climate, etc.,

- influences such as persons, experiences, etc., and

- learned outcomes.

Initial findings and insights from the interview data related to students' definitions of ethics generally and engineering ethics specifically and their perceptions of learned values and outcomes associated with ethics and social responsibility are also included. As additional context and background for this baseline design phase, the methods section of this paper describes data collection and analysis procedures for these preliminary results.

\section{Background and Literature Review}

A large portion of the literature in engineering education related to ethics has focused on content and pedagogy and general ethical (or unethical) behaviors such as cheating or participation in pro-social activities ${ }^{2,3,4,5,6}$. However, an increasing number of studies are investigating foundational considerations such as student levels of moral development or student perceptions of, and attitudes toward, social and ethical responsibility. Holsapple and colleagues concluded that students often have relatively narrow understandings of what qualifies as ethics compared to the faculties' beliefs in how ethics has been taught and demonstrated. ${ }^{7}$ Loui explored students' perceptions of the characteristics and responsibilities of professional engineers as related to their professional identities. ${ }^{8}$ Indeed, Zoltowski and colleagues found that students often struggled to identify "everyday" ethical considerations or issues within their design considerations or within their team processes. ${ }^{9,10}$ Canney and Bielefeldt have developed a model of personal and professional social responsibility development, as well as the relationship between professionals understanding of social responsibility and their perception of ethical dilemmas. ${ }^{11,12}$

Additionally, the larger literature based on organizational ethical climate describes how social norms and culture can influence individuals' behavior. For example, Rudnicka showed how “contextual/environmental factors” affect students’ ethical reasoning and decision-making abilities. Specifically, factors such as team learning dynamics, work experience, the culture of the engineering field, and the moral intensity of a given dilemma or situation play important roles in decision-making. ${ }^{13}$ In their qualitative study of relationships between individual ethical understandings and team ethical climate, Feister and colleagues found evidence that students' ethical perceptions and decisions differed based in part on programmatic orientations (e.g., entrepreneurship, business, and community engagement. ${ }^{14}$ In addition, their study of a servicelearning design program found that although a human-centered design program utilized in the program was helpful in helping students orient to ethical considerations, motives, and decisions, the students tended to identify only ethical concerns linked to interactions with users and still struggled to explicitly identify ethics and articulate its role in their day-to-day design work. ${ }^{15}$ Sunderland adds curricular approaches and constraints as important contributing factors, narrowing students' ethical perceptions. For instance, students perceived educational curricular reforms that present increased social and ethical requirements as "constraints" and "discrete requirements" rather than integral to their education and future professional practice. ${ }^{16}$ 
Looking beyond the engineering education field, qualitative studies of professional ethics have been frequently carried out in the medical field. These studies have many parallels to the current work in engineering ethics. For example, a 2005 review article on medical ethics education identified deficits in the literature in the areas of the theory of medication ethics education, empirical studies on student outcomes, and studies on the pedagogy of medical ethics education. ${ }^{17}$ This study pointed out two points of view regarding the goal of medical ethics education: that it is a way to create virtuous physicians, or that it is a means of providing physicians with the skillset they need to approach ethical dilemmas. A 2002 study of nursing also focused on virtue ethics. This work analyzed open-ended survey responses from 53 registered nurses about what makes a good nurse and how a nurse does the right thing (Smith and Godfrey, 2002). ${ }^{18}$ This was in part a response to a tension in medical ethics of whether nursing should subscribe to an ethic of justice or an ethic of care.

\section{Methods}

\section{Data Collection}

As part of the baseline phase of data collection, a survey was administered to determine undergraduate engineering students' perceptions of ethics generally and for engineers, social responsibility, and other related constructs, as well as students' demographic information. This survey was administered to freshman engineering students at four universities, Arizona State University (ASU), Brigham Young University (BYU), Colorado School of Mines (Mines), and Purdue University (Purdue). A total of 780 students completed the survey and the results of the analysis of that data can be found in a previous conference paper ${ }^{1}$.

The demographic data from that survey was utilized to stratify the selection of follow-up interviewees as part of baseline data collection. Students were selected based on gender, major, international status, and age for longitudinal purposes described more fully in the participant demographics section of this paper. Stratification resulted in approximately 30 completed interviews from each institution.

An interview protocol was developed that would provide insight into four areas of inquiry: (a) general definitions (including macro-ethics), (b) experiences that influence ethical perceptions past, present, and future (including justice), (c) ethical climate at the students' undergraduate institution, and (d) elaboration on the ethical scenarios and associated responses included in the survey. Pilot interviews were conducted with a few of the students selected through the stratification process. Approximately three pilot interviews were conducted at each institution, attending to a variety of interviewee demographic characteristics. It was determined after the pilot interviews that the questions should be reordered to flow more smoothly. Also, a couple of clarifying examples were added for questions students didn't clearly interpret.

After revision of the interview protocol based upon pilot interview feedback, the protocol remained the same for all interviews conducted across the institutions. However, because of the semi-structured nature of the protocol, interviewers asked various follow-up questions to better understand students' ethical perceptions, influencing experiences, etc. 
Each interview was transcribed into data that was coded in NVivo through an iterative, multirater, multi-institutional process. NVivo is a software program that allows multiple viewers of the same transcription, which can then be merged into one coded document. Reports were then compiled of the various codes for further analysis as described in phase two of the qualitative analysis. Although the amount of data and cross-institutional analysis involved in this study posed its challenges, multiple perspectives have provided ample revisions to coding frameworks and robust analysis of interview transcripts. In an attempt to consider the perceptions of ethics of the 113 interviewees, a subgroup of 29 interviews were selected based on similar stratification criteria as the interview selections. These 29 interviews provide the data for the current analyses. The researchers intend to continue to analyze the remaining interviews in the future.

\section{General Interviewee Demographics}

The interviewee subgroup, comprised of 29 interviews, is representative of the larger study population in general characteristics. In the subgroup, there were 10 female students (34\%), and 19 male students (66\%). These percentages are approximately equal to the gender distribution of all the survey participants with 31\% female and 69\% male across the four collaborating universities. These distributions are slightly higher than the national engineering student population of approximately 18-20\% female students compared to male students ${ }^{19}$.

In the interviewee subgroup, there are three international students, comprising $10 \%$ of the subgroup - one female from Purdue, one male from BYU, and one Male from Purdue. This distribution is much higher than the participant population as we intended to oversample foreign students due to possible attrition and lack of representation across the longitudinal study. The entire study population is comprised of approximately $5.4 \%$ international students.

The distribution of interviewees was fairly representative of the study population with four students from ASU (14\%), nine students from BYU (31\%), seven students from Mines (24 \%), and nine students from Purdue (31\%). The mean ages of both the interviewee subgroup as well as the overall study participants is 18.4 years old. Table 1 shows the racial/ethnic breakdown of engineering student participants. Three participants marked mixed race on their survey responses, meaning they indicated multiple races for the same person, resulting in greater than $100 \%$ for overall race. One participant did not indicate race.

Table 1 - Interviewee subgroup breakdown by race

\begin{tabular}{llr}
\hline Race/Ethnicity & Frequency & Percent of Subgroup \\
\hline American Indian or Alaska Native* & 1 & $3.45 \%$ \\
Asian or Asian American & 4 & $13.79 \%$ \\
Black or African American & 0 & $0.00 \%$ \\
Hispanic or Latino/a* & 3 & $10.34 \%$ \\
Native Hawaiian or Other Pacific Islander & 0 & $0.00 \%$ \\
White or Caucasian* & 23 & $79.31 \%$ \\
Other* & 2 & $6.90 \%$ \\
\hline Total* & 33 & $113.79 \%$ \\
\hline
\end{tabular}

*Mixed race/ethnicity reported. 
While religion is not necessarily an indication of ethical or moral disposition, the survey results of this study suggested a significant correlation between the importance of religion and increased ethical knowledge and beliefs. ${ }^{18}$ In other words, students who reported religion as important in their lives scored higher on the measures of ethics. Given that one of the universities involved in the study is a private religious institution, the researchers are interested to consider whether the student population there varies in their ethical considerations from the other institutions in the study, or if particular activities and programs affiliated with that institution have varying impacts from ethics activities at the other schools. The religious makeup of the interviewees is listed in Table 2. While other options of religion were available to select on the survey instrument, only those indicated by the interviewees are listed in the table.

Table 2 - Religious affiliation of interviewees

\begin{tabular}{lll}
\hline Religion & Frequency & Percent of Subgroup \\
\hline Atheist or agnostic & 7 & $24.14 \%$ \\
Christian & 17 & $58.62 \%$ \\
Hindu & 2 & $6.90 \%$ \\
None & 2 & $6.90 \%$ \\
Other & 1 & $3.45 \%$ \\
\hline
\end{tabular}

There are a wide variety of majors available to engineering students at the four participating universities. There is not a dominating major or minor for the interviewees in the subgroup, with the highest percentage of students reporting Mechanical Engineering as their intended major (27\%). Civil Engineering and Materials Engineering are both represented by $11 \%$ of the students. The percentage of civil engineering students in the subgroup is slightly higher than that of the overall study student population. Civil engineering students were purposefully oversampled to determine the effects of the ethics education curriculum for civil engineers. Considering the longitudinal nature of this study, it was determined by the researchers that an effective way to consider the effects of the required ethics education courses for civil engineers was to ensure sufficient civil engineering students in the culminating interview process. Attending to attrition, it was determined oversampling of civil engineers at the baseline data collection phase would support investigation of this hypothesis.

Table 3 outlines the intended major reported by the students in the interviewee subgroup. These are only the majors reported by this subgroup of participants. This list is not comprehensive of the majors reported by all of the participants of this study, nor all of the possible engineering majors at the four universities. There are 44 listed majors for only 29 participants, indicating that some participants in the subgroup listed dual majors or engineering minors as majors. Percentages were calculated out of 44 possible majors, not out of 29 participants.

Students in the subgroup reported a wide variety of minor programs of study, some of which were engineering and STEM related while others were not. Several reported minors in the arts. 
Table 3 - Intended major and minor engineering programs of study reported by the interviewee subgroup.

\begin{tabular}{lrr}
\hline Major & Frequency & $\begin{array}{r}\text { Percent } \\
\text { of Subgroup }\end{array}$ \\
\hline Mechanical or Mechanical Systems Engineering & 12 & $27.27 \%$ \\
Civil and Environmental Engineering & 5 & $11.36 \%$ \\
Metallurgical and Materials Engineering & 5 & $11.36 \%$ \\
Chemical or Biochemical Engineering & 4 & $9.09 \%$ \\
Aerospace, Aeronautical, or Astronautical Engineering & 3 & $6.82 \%$ \\
Other Engineering Major (2 or fewer students per major) & 15 & $34.09 \%$ \\
\hline Total & $\mathbf{4 4}^{*}$ & $\mathbf{1 0 0 \%}$ \\
\hline
\end{tabular}

\section{Political and Social Involvement Scale}

On measures of political and social involvement, the interview subgroup is generally representative of the sample population as a whole. Nearly all of the interviewees indicated a focus on improving society. However, influencing the political structure was one of lesser interest. Therefore, this group seems to have a high societal focus while maintaining a low political interest, as can be seen in Table 4 . The preliminary survey results highly correlated ethics scores with religious importance ${ }^{18}$ in both the interview subgroup, as well as the sample population, approximately half the respondents indicated religion and spirituality are important to them. Thus, the interview population is representative of the sample population related to this key aspect.

Table 4 - Political and Social Involvement Subscale of interviewees.

\begin{tabular}{|l|l|l|l|}
\hline Item & Topic & $\begin{array}{l}\text { Subgroup Percent } \\
\text { Positive (3 or 4) }\end{array}$ & $\begin{array}{l}\text { Sample Percent } \\
\text { Positive (3 or 4) }\end{array}$ \\
\hline $34-9$ & Influencing the political structure & $37.9 \%$ & $31.0 \%$ \\
\hline $34-1$ & Becoming a community leader & $48.3 \%$ & $48.3 \%$ \\
\hline $34-10$ & Integrating spirituality into my life & $51.7 \%$ & $57.1 \%$ \\
\hline $34-2$ & $\begin{array}{l}\text { Becoming involved with activities that } \\
\text { preserve and enrich the environment }\end{array}$ & $55.2 \%$ & $58.8 \%$ \\
\hline $34-5$ & $\begin{array}{l}\text { Keeping up to date with political } \\
\text { affairs }\end{array}$ & $62.1 \%$ & $42.8 \%$ \\
\hline $34-7$ & $\begin{array}{l}\text { Helping to promote racial } \\
\text { understanding }\end{array}$ & $62.1 \%$ & $52.6 \%$ \\
\hline $1-12$ & How important is religion in your life? & $62.1 \%$ & $61.5 \%$ \\
\hline $34-4$ & $\begin{array}{l}\text { Improving my understanding of other } \\
\text { countries and cultures }\end{array}$ & $69.0 \%$ & $60.9 \%$ \\
\hline $34-8$ & Influencing social values & $69.0 \%$ & $61.9 \%$ \\
\hline $34-11$ & Volunteering in my community & $69.0 \%$ & $68.2 \%$ \\
\hline $34-6$ & $\begin{array}{l}\text { Developing a meaningful philosophy } \\
\text { of life }\end{array}$ & $75.9 \%$ & $70.4 \%$ \\
\hline $34-3$ & Helping others who are in difficulty & $82.8 \%$ & $82.3 \%$ \\
\hline $34-12$ & Improving society & $93.1 \%$ & $87.7 \%$ \\
\hline
\end{tabular}


As part of the survey, students were given a list of potential activities and asked to indicate which they had participated in during the last five years or intend to participate in during the next five years. Part of the interview protocol asked follow-up questions about the experiences indicated in the survey. To understand some of the previous experiences indicated by the students in the interviewee subgroup, the most prevalent are listed here, followed by the percent of the subgroup that indicated past participation in that activity: (a) volunteered regularly (79\%), (b) participated in honors programs (59\%), (c) participated in formal religious instruction (58\%), (d) traveled or lived in a developing country or region (41\%), (e) mission or volunteering trip (38\%), and (f) participated in formal ethics instruction (38\%).

\section{Qualitative Analysis Methodology}

To begin the analysis of the interview transcripts, ten interviews were considered by a group of three to four raters from the various participating institutions. This group created a codebook based on emergent themes, relying heavily on the organization of the interview protocol. After multiple iterations of coding and consulting with the entire research group, they determined three general areas of focus: (a) perspectives of ethics, (b) experiences students mentioned have influenced their ethical perceptions, and (c) outcomes learned through those experiences. Once the codebook was created, it was used to similarly code the next ten interviews in the first subgroup. Again, multiple iterations across raters and institutions led to a refined codebook that was then used to code the remaining 9 interviews, for a total of 29 interviews in the subgroup reported here.

The codes attending to students' perceptions of ethics were divided into general definitions and perspectives specifically oriented toward engineering ethics and professional identity of engineers. The general definitions include questions from the protocol prompting for attributes of ethical and moral character, examples of ethical disengagement, justice related situations, personal goals, and institutional climate, among others. Engineering identity and questions related to workplace dilemmas, as well as reasoning about ethical scenarios specific to engineering are among the responses coded specifically for perceptions of engineering ethics.

The experiences students mentioned as having influenced their ethical perceptions were coded for common themes. In the first group of ten interviews, it was determined these experiences would be coded to answer Research Question 2, which attempts to consider the types of experiences that are influential during the engineering undergraduate program. However, it was also determined that the experiences needed to be clustered in ways that would facilitate analysis and interpretation of these experiences, considering the large amount of data collected in this study. The influencing experiences were clustered according to the type of experience as follows: (a) academic, (b) extracurricular, (c) family, (d) international, (e) professional or work related, (f) religious, (g) service or volunteer oriented, and (h) social or affected by friendships. Each of these categories was clearly defined and examples of each were provided for coders. Through multiple iterations and in attending to subsequent analysis of transcripts, it was determined that these categories were exhaustive and one or more of these categories could identify all of the experiences related by students. 
The code for learned outcomes is inclusive in that all of the learned outcomes were combined together, rather than distinguishing between outcomes learned from general experiences as compared to engineering-related experiences. This was done because most of the freshmen students don't have previous engineering experience, or at least not sufficient to create patterns that would lead to learned outcomes. Therefore, the learned outcomes code includes statements of discovery of lessons learned and the morals of lived experiences reported by students during the interviews.

After initial coding into various perceptions, influences, and learned outcomes, it was determined that for this paper, general definitions of ethics, engineering identity, and learned outcomes would be further analyzed to consider common emergent themes within each and across these three codes. It was also determined that the frequencies of influential experiences would be better portrayed at a later date, once more of the interviews were coded and included to better represent the study population. Likewise, other aspects of students' ethical perceptions will be further analyzed in the future. The analysis of this phase of coding is presented in the findings section of this paper.

\section{Findings}

As described in the methods section, perceptions of ethical and moral character, engineering identity, and learned outcomes data were further analyzed. The findings from each of these codes are described individually in this section. The discussion section of this paper discusses how these themes are related and illuminates implications pertaining to some or all three of these categories.

\section{Perceptions of ethical and moral character}

Inductive analysis of responses coded as "Definitions of ethical character" led the researchers to identify three main clusters of responses in the data set: 1) general rules/norms, 2) other-oriented statements, and 3) specific norms or traits. The themes and associated sub-themes are summarized in Table 5. The narrative that follows emphasizes themes that were discussed by at least two interviewees.

Table 5 - Overview of emergent themes for general definitions of ethical/moral character

\begin{tabular}{|l|l|}
\hline Theme & Sub-Themes \\
\hline \multirow{4}{*}{ 1. Rule/norm-based } & a. General (doing what is “right" or "best") \\
\cline { 2 - 2 } & b. Right or best for self \\
\cline { 2 - 2 } & c. Right or best for society \\
\cline { 2 - 2 } & d. Differences - Individual, contextual, situational \\
\hline \multirow{2}{*}{ 2. Other-oriented } & a. Helping or caring for others \\
\cline { 2 - 2 } & b. Consequences/impacts on others \\
\cline { 2 - 2 } & c. Sacrifice of self for others \\
\hline \multirow{3}{*}{ 3. Specific norms and traits } & a. Norms \\
\cline { 2 - 2 } & b. Traits \\
\hline
\end{tabular}


As additional framing for the write-up that follows, it is worth starting with a more general observation about the ability of students to distinguish ethics from morality. On the one hand, it is notable that at least one interviewee was able to provide a fairly nuanced comparison of the two concepts, describing morality as "more of a personal thing" and ethics as "more of a codified standard." On the other hand, only a few students even attempted this delineation, and those who did typically fell short of a satisfactory response. The results that follow should therefore be read and interpreted as reflecting how student subjects typically understand ethics, morality, and related concepts rather than how these terms are more formally or technically defined.

Rule/norm-based. The first major theme in the findings is comprised of those statements characterizing ethical or moral character as involving adherence to rules or norms in general. Approximately two-thirds of the interviewees made comments falling in this broad category, with the most common and most general type concerned with knowing and/or doing what is "right” or "best." Representative examples of this type of statement include "to understand comparatively what is truly right," "doing the right thing”, and "making the right decision." A subset of this group additionally stressed the importance of doing right even if faced with worrisome or troubling consequences. For instance, one student framed this as "choos[ing] the right action regardless of consequences", while another explained that someone exemplifying moral character would make "the morally right choices even if those choices may not actually be in their best interest."

A smaller number of interviewees were more nuanced about the source of rules or norms that might guide such decision-making. One group of students explicitly emphasized the importance of adhering to one's own principles or values, e.g., "living up to your standards" or "what you personally believe is right.” Another group of respondents acknowledged society as an important source of ethical or moral guidance. For instance, some interviewees were explicit in pointing to the importance of "following rules and laws and stuff like that", while others more generally referred to "praiseworthy behavior as a societal view of it" or "how your actions are acceptable to the greater society."

At least one interviewee additionally acknowledged potential tensions between individual and societal norms, referring to the "standards you put for yourself, usually according to society, that you intentionally or unintentionally follow or don't follow.” On a related note, at least six students acknowledged situational, contextual, and/or individual variations in ethics or morality. For instance, one student explained how her experience studying abroad in Germany "taught me that morals are, in many cases, societal which I think really freaked me out at the time." And as another added: "ethics vary by culture and by person and kind of depends on how you're raised."

It is interesting to note that eleven participants from the private religious institution made statements regarding rule or norm-based ethical perceptions, as compared to four, six, and eight from the other three universities. This may be attributable to the strict honor code of the university or the necessary adherence to religious standards perceived by students from that particular school. One student from that institution even associated ethics with religion when stating, "I also feel like moral character is mostly influenced, in our society at least, by religion. What God thinks is right is what we should be living, but not everybody necessarily does that." 
Other-oriented. Statements related to a second major theme, which is described as "otheroriented", were found in more than two thirds of the transcripts. Three prominent subthemes were identified in this cluster. The first of these involves comments about "helping" or "caring" for others, most often in explanations for why students picked specific individuals as moral exemplars. For instance, one interviewee framed television personality Fred Rogers ("Mr. Rogers") as someone who "actually cared about other people," while another identified President Obama as someone who "help[s] people." Yet another student described how football star Peyton Manning "gives back to others.”

A few of these students offered even more complex interpretations, including by describing special obligations associated with caring or helping. For instance, one student noted "a moral responsibility to help those that are less off when you are in a position of power." And the same interviewee who cited President Obama as a moral exemplar similarly declared that "more power comes with more responsibility." One interviewee approached the matter from an alternate angle by naming Native American rights activist Ramona Bennett as her exemplar. As she explained:

"Part of morality and ethics is knowing that you're guilty in some way. It's like knowing you're guilty of perpetuating the system that you might not have known even existed for a lot of your life, like racism or something. A really big part of, I think, becoming an ethical person is recognizing that guilt, and moving past it to help those who are oppressed or who are wronged or acted immorally against.”

While this passage suggests a strong sense of awareness for issues of privilege and justice, this was the only interviewee who offered such an articulate and nuanced view of "helping." Yet there were many students who did speak more generally about how helping or caring for others can benefit society or humanity. For instance, one student presented examples of people helping others through philanthropic work as "improving the human condition," while another characterized moral or ethical character as "anything that helps the betterment of society."

A second variation of the "other-oriented" theme involves statements about the consequences or impacts of one's actions. This subtheme was reflected in one student's portrayal of ethics as in part concerned with "taking into account other humans" and another's comment about how one's actions "benefit others." One interviewee additionally shared a relevant example about dividing up a scarce water supply during a desert backpacking trip, concluding: "you really do have to consider how it's [a difficult decision] going to affect other people and the way that you act.”

Two additional variants of this subtheme are worth noting. One variant involves statements paraphrasing the "Golden Rule”. In describing his mom as a moral exemplar, for instance, an interviewee noted that it was important to "treat other people and treat society how you would want to be treated yourself." There were two statements of this sort in the data set. A second variant can be found in a student's comment that "the ethical character of a person is mostly defined by what they believe to be either harmful or beneficial to other people and themselves", as well as another's framing of moral decisions as "the least harmful out of possibilities." These were the only instances that were coded that explicitly mentioned consideration or reduction of harms. 
A third notable subtheme centers on statements about sacrifice, many of which appeared in discussions of moral exemplars. For instance, a student described George Washington as a moral exemplar who "put the need of the people before the need of himself." A number of other students described close relatives in a similar manner, with one noting how his dad was known for "doing things for us rather than himself", another interviewee describing his grandfather as "very selfless ... aware of our needs rather than his own", and still another portraying a more senior peer as "not interested in bettering himself as much as he is the community around him."

Specific norms and traits. The preceding section also hints at specific characteristics sometimes attributed to moral or ethical individuals, e.g., selflessness. Indeed, the third major cluster of statements in the data set is comprised of specific norms and traits that were explicitly mentioned by interviewees. To begin, a number of students pointed to specific behaviors in normative terms. For example, some students described their moral exemplars as the type of people who did not cheat, lie, and/or steal. Another student described his dad as a moral exemplar who "always does the best he can," while another pointed to "working hard" as a desirable trait. At least three students additionally noted the importance of fairly or generously distributing credit, as reflected in statements such as "taking credit for your own work" and "giving credit to the person that deserves it."

Regarding specific traits, about a third of interviewees made comments about honesty, integrity, and related concepts. These range from simple mentions of "honesty", "integrity", and "trustworthy" as traits associated with moral exemplars. More specific and nuanced formulations can also be found, such as one student's comment about "always being honest in your work and not falsifying results" and another's depiction of someone who is "honest in their dealings with everyone, both those above them, both those below them, wherever they're standing." Other subjects made complimentary remarks about accountability, as in one student's discussion of ethical and moral character as "being honest in everything that I do, you're accountable for your actions" and another's framing of ethics as being "responsible for what you do to society." And complimentary to the "other-oriented" themes discussed above, multiple students additionally alluded to selflessness, e.g., having a "selfless spirit” or "you're just not all about yourself.” A final example of a specific trait can be found in comments related to empathy, with one student explicitly proposing a connection between emotions and ethical decision making, and another noting the importance of having "social awareness ... in that other people have feelings too."

A final cluster of traits was more overarching or cross-cutting in character. For instance, at least three students pointed to the importance of one's "conscience" in relation to ethics and morality, with one explicitly defining ethical or moral character as "someone who strives to have a clear conscience." Other students emphasized the need for moral fortitude, as in references to "sound or unmoving morals," "morally strong people," or being "steadfast" in the face of ethical or moral dilemmas. Two more student subjects made statements about moral courage, namely by describing the need "to just be self-aware and to disagree when you feel like you truly need to disagree" or "to accept that you will have to deviate from the group at times and it will suck." 


\section{Engineering Identity and Ethics}

It is important to note that all names indicated are fictitious and were assigned to de-identify any student participants. Inductive analysis of responses coded as "Engineering Identity" yielded themes that were similar to general understandings of ethics and morality, but yet were distinct.

Norms/Traits. As with general ethics, honesty and integrity were cited by a number of interviewees as important characteristics for an engineer to possess. An example response is given by Beverly:

"I think honesty is a big one. I think at any field, not just engineering, honesty is the best quality as they say. It's really important, because as engineers you're making all these decisions that impact not just you but thousands and millions of people. You have to make those honest decisions, because they could be the deal breaker or they could be that one thing that makes the bridge collapse or holds it up for a lifetime."

Other specific and general traits that were in common with the students' definitions of ethical/moral character included being humble, open-minded, and personable, and having respect, empathy, and self-awareness. However, the interviewees identified some unique traits related to the engineering ethics context, such as having common sense, hardworking, diligent, and loyal.

Technical competence was the most common trait identified by the students that was unique to the engineering context. Some of the interviewees who stated technical competence as important for engineers to possess also demonstrated an awareness of the impact and power of the specialized skills that engineers possess, and thus, the responsibility they have to protect general public.

The interviewees also referenced engineering norms such as ethical codes, building codes, and standards when asked about the professional responsibilities. In these responses, the students specifically cited aspects of codes of ethics such as holding safety paramount, not working outside of expertise, and only signing drawings if they are okay. As related to standards, the students described being taught how to do wiring or milling correctly or the importance of tolerances. For example, Carl stated:

"As for the ethical portion of that, I think engineers have a responsibility to consider all aspects of what their project is affecting. In some cases, that requires engineers of different disciplines to make sure, "Hey, this isn't going to be a problem," or whatever, because just different areas of expertise. That just makes sure that all work that's being done is safe for the community. As for the professionalism part, just keeping your work straight, signing off on things that you should, and not signing off on a blueprint that doesn't look very good. That's not going to work, so you shouldn't sign off on it.”

Another skill that was identified by over half of the interviewees as being important for ethical engineers to possess was communication. The importance of this skill was described in primarily two different contexts: 1 ) the responsibility of engineers to communicate potential problems or concerns to appropriate persons, and if needed, go to the public with the information; and 2) the 
responsibility to communicate complex technical information to non-technical audiences in a way that they can understand, and not to use the technical language to their advantage. An example of the later is a response given by Corvin:

"In terms of interactions, a lot of people become engineers because they like the math and science, and they may not be necessarily the best writers or the best communicators. You still have to understand that as an engineer you're going to have to communicate what you're doing with other people who are non-engineers. It's not practical to throw a bunch of extremely technical designs and words at people who don't understand them.”

Impact. Many described a need to have an awareness of the "impact" on broad stakeholders or entities, such as society, the public, the environment, and safety, without further defining what is meant by impact, or the complexity of the considerations and issues involved in this awareness. An example of this description of impact is given by Petunia:

"I think it goes back to thinking of others because you're manufacturing or creating things that have impact on a large number of people and you need to make sure that you're holding their safety paramount and also, we talked about the code of ethics in our class.”

For many of the interviewees, the primary responsibility of engineers is beneficence, which was also reflected as a responsibility in their general descriptions of ethics. Within the engineering contexts, these statements included those such as “do good," "help," "make lives easier," and "positive impact on society," but also expanded those to include "safety” in their descriptions of beneficent actions. For example, when asked what professional responsibilities and obligations engineers have to society, Adam responded:

"Number one has to be to keep them safe. I think that's the whole reason why engineers do what they do is to help make society a better place, so I think their first obligation should be those that they help. To keep them safe and prove the environment for those that they work for."

Although both the general and engineering ethics responses reflected prioritization of beneficence, discussions of non-maleficence were found almost exclusively in the engineering ethics responses. The students described the responsibility to "do no harm" nearly as frequently as beneficence, and the two principles often mentioned together. For example, Andrew described engineers' professional responsibilities and obligations to society as:

"They always have to keep in mind that their work can't harm society or do something in a negative way. It always has to impact society in a positive way, like help other people out.”

In the descriptions of "impact" in both the general and engineering ethics, the interviewees referenced entities such as the public and society as to whom they were responsible. When describing the responsibilities of engineers, the environment and safety were also included as illustrated by the following response by Chase: 
"Again, they're in charge of the safety of people, like if something goes wrong, structurally or like the British Petroleum oil spill, that was also an engineer's fault. That has poor repercussions on both the environment and people, so they just got to protect everything."

Understanding of the Considerations. As illustrated by many of the quotes in the previous section, many of the interviewees described broad priorities, but did not articulate an understanding that reflects the complexity and interrelatedness of these priorities. However, some students did describe different, competing elements, or unintended consequences, related to the ethical and social responsibilities of engineers. In these descriptions, the interviewees included a variety of considerations, such as prioritizing the stakeholder, usability, the "needs" of the user or project, and quality. Consideration of these aspects often was done in the context of design, as illustrated by Paula's response:

"I would think stuff like, if you're designing a product or something, where the materials are coming from, is that going to, is this something that's going to cause issues, or it's going to be really damaging to the planet or something. How people use it, is there going to be any major safety risks, and how can you minimize that or like make it really clear, like, "Hey, this is a saw, it's sharp. Be careful," and then ..."

One of the most common examples of competing objectives given by the students were ethical issues related to the objective to reduce cost and the negative impact it could have on safety. In addition, the students identified actual and hypothetical situations where engineers faced or might face business and economic pressures to make unethical choices related to safety to falsification of data or shortcuts taken by the engineers. Another example of how students considered multiple issues is a response by Brody, who described the ethical issues which arose from the unintended consequences of an initiative to reduce emissions:

"One of the most recent lectures that I went to, I'm not sure if you're aware of it, but there was an engineer that spoke here for all the mechanical engineering students or I guess all the engineering students. He talked about a port that they're building in California that is completely electric and releases no emissions but there was a problem with the noise pollution factor. I don't remember what it was. It affected hundreds of households nearby. It was something that, although it was reducing emissions, is it still right to affect all these people living nearby because they didn't really choose to put it there.”

A limited number of the interviewees described more complex integrations of multiple issues, including of how they considered the stakeholders. For example, Christopher's awareness of the interconnectedness of the stakeholders informed his understanding of the impact of their design:

"It gave me a new insight to the vast [pause] I guess I had a very basic knowledge of how engineering and actions impact others but it made me more aware of the true web of stakeholders, as they call them, that are affected by any action or product or any solution. Because for our project we have to find a way to process or remove ocean plastics or plastics on the beach. Difficult task and a lot of stakeholders involved. Basically, all of the human race and biosphere but more specifically per region.” 
Another interviewee, Corvin, demonstrated a nuanced understanding of the integration and complexity of the issues, and taking in account the cultural considerations, in his design:

"Then in terms of just designing you also, as an engineer you have to look through the side effects of what you're doing both as an environmental standpoint which is a big push these days, but also as a cultural standpoint. I'm in the Grand Challenges pilot course here. In that, we're designing water filters for homes in the Ganges river valley. When we're designing those, we have to take into account the culture of the area and that to them the river is a sacred being. It's a deity basically so you can't alter the river.

When we were looking at, "Okay, how can we fix this problem, is there a way to fix it on a bigger scale than just a simple water filter?” We looked at cleaning the river but as a culture, they don't want to mess with the river because the river is a deity. That led us to the point where we have to take the water out of the river before we purify it in any way. You have to take those cultural considerations into account as well.”

Relationship of engineer to society and to employer. The students' understanding of the ethical and social responsibility of engineers was also discussed in relation to other entities. In the interview, the students were asked to explain their responses (on a scale of strongly agree to strongly disagree) to the following survey item: "Surprising and risky uses or new technology, such as social networking websites, are completely the responsibly of those who use them." Most of the students indicated that engineers and users have shared responsibility. Many also indicated that they could not control (i.e., it wasn't their responsibility) if users misused the technology, with some of those going further to say that engineers have a responsibility to consider, and prevent as much as possible, incorrect or harmful ways that technology could be misused. A limited number indicated that the users assume all of the responsibility, but that may be a result of providing the social networking example, a topic which the students might see as unique among engineering products.

When asked about their responsibility to their employer, the engineering specific traits described earlier were often identified (e.g. hardworking, diligent, loyal). They often described the importance of respecting their employer, but also recognized their responsibility to their self and the public above responsibility to employers. A few students stressed the importance of doing work that aligns with personal ethics, and recommended finding other work if it does not. Most all of the interviewees recognized the tremendous responsibility of engineers, as shown in Pete's response for what it means for engineers to be ethical or have high levels of professional integrity:

"Well, it's engineering. It's not like [pause] when you make a mistake, it's not like mathematics where you [pause] maybe something doesn't work out right, you can always go back and fix it. You may actually risk people's lives when you make a mistake. So, our choices are heavily weighted.” 


\section{Learned Outcomes}

During the interviews, students frequently offered explicit or implicit statements about insights, learnings, or realizations related to ethics which they gained from an experience or situation. These learned outcomes were coded using an emergent coding process. Examples of the learned outcomes mentioned in the 29 interviews being analyzed are presented in the following sections.

Learned Traits or Values. A number of interviewees described traits or values which they learned were important to ethical decision-making. These included empathy, responsibility, good work habits, and honesty. One of the most frequently stated learned outcomes was focused on the role of empathy in ethics. Such observations generally could be divided into two subcategories: empathy for the viewpoints, feelings and experiences of others, broadly, and empathy for the ethical behaviors or decisions that others make. As an example of gaining empathy generally, one student discussed writing an essay in high school about a gay family member which, "opened [her] eyes to stuff that [she] didn't realize before, stuff that happened, the way that people are treated." Another student described a leadership experience which taught him, "It's important to be able to ... see from someone else's perspective into [sic] order to make a decision that will be best for [the] general populous.” The interviewees frequently cited that they developed empathy through a situation such as traveling or living abroad, interacting with others different from themselves, volunteering, or a human-centered design course.

In the other subcategory, the interviewees said that empathy helped them understand why another person's ethical views were not aligned with their own and why a person might make an ethical decision that they disagreed with. After describing one experience, an interviewee said, "I realized, what if people act this way or act different ways because of this situation? I never thought about that before.” As quoted in the discussion of perceptions of ethical character, a student said that she learned,

"Morals are, in many cases, societal which I think really freaked me out at the time. I'm realizing there's a use for it... I think, again, there's also that autonomy you have to maintain. You have to realize, Okay, my society and my country might think this but I think this."

Nearly a quarter of the interviewees connected fulfilling one's responsibility to ethical behavior, citing cases where they or people they knew were not living up to their responsibilities as citizens, employees, teammates, or students. For example, one student, Bobby, presented a situation where his co-workers hadn't completed their assigned work. He explained,

"I didn't have to report to my supervisor, but I had to make the choice to talk to my supervisor and let her know about the work that hadn't been accomplished by these other employees. That was kind of tough because you don't want to ever rat anybody out or anything, but it's also part of their responsibility and it hadn't gotten done...”

Another student described a time when he was asked by an employer to do something that pushed him out of his comfort zone (going on stage during a theatre production while serving as the stage manager), and the student asked someone else to do the task because he was 
uncomfortable being on stage. The student explained, "Since then I always thought, responsibility is more important than your own personal feelings as long as what you're doing is right. There's nothing wrong that I would've done or I could've done if I'd gone on stage and just done what I was asked. What I didn't do caused me to be wrong.”

Students also pointed to work habits such as working hard and being motivated as playing a role in ethical behavior. For example, one student identified procrastination as an ethical situation, citing a case where her procrastination negatively impacted her group, and a second case where her procrastination nearly led to a case of academic dishonesty. Another student shared the importance of completing high quality work, having learned that, "good quality work doesn't happen overnight,” and that, "you've got to work really hard on it.”

Finally, interviewees also frequently cite honesty as a trait which they learned the importance of. The student, Bobby, referenced above whose coworkers had not completed their work explained, "I think I learned it's always just better to be honest with the supervisor or with an employer because you don't want that to ever come back to you. When you aren't at first at fault, it could come back and then you could end up being at fault.” Another student who worked as a door-todoor salesman explained how being dishonest was unethical in his job:

"When you're selling, you're trying to make sure that when they get the product, it's everything that you told them it's going to be, not over sell it, don't tell them like, "I sold pest control so you're not going to see a single bug in your house. I promise you." That's impossible, unethical.”

Learned Behaviors or Behavioral-Change. Students often offered a specific behavior that they learned from a situation, or a behavior which they learned to not engage in after an experience. The most frequently addressed behavior was academic or professional misconduct, but interviewees also touched on the importance of communication, the personal treatment of others, and teamwork.

Students frequently discussed situations of misconduct (professional or academic) as both an experience which they found to be ethically challenging and one from which they gained certain realizations. One student described in-class discussions in high school, where the message was conveyed that, "you should always fail with your ideas rather than succeed... by taking credit for someone else's ideas." Students would often cite that, following an instance of academic misconduct, they learned to never engage in a similar unethical behavior (copying a peer's homework, allowing someone to copy their work, plagiarizing, making up data) again.

Students also discussed a theme related to learning the importance of communication in potentially ethically-fraught situations, learning to work with others, and learning to resolve interpersonal conflicts. One student discussed an in-class design scenario where,

"You were supposed to get some sort of nuts and bolts for this part of the project and it wasn't available locally, but they wanted all parts to be local. If you wanted that specific nut or bolt or whatever, you had to import from somewhere else. It's like, you can import from somewhere else, so you're 
meeting one part of the specification that you're getting that exact part for them, but it's not locally produced, so that's going around [sic] the word. Rather than sneaking behind their back and getting it from somewhere outside, or getting probably low grade parts for them locally, you could speak to them and be like, 'Hey, are you ready to compromise on this part, or do want something that's of cheaper quality from locally?'”

Another student explained that they found a mentor to be especially ethical, since the mentor always treated others kindly, even if the other person was a stranger or not nice to him. Students frequently cited the importance of teamwork. One student explained that, after a series of in-class activities focused on teamwork, "we kind of realized as we were doing the activities that we couldn't just do things on our own." Another described learning through a themedlearning community in her freshman dorm about engineering problems that,

"Problems are interconnected and how you don't need to be a philanthropist or a humanitarian or a politician or some really influential figure in society to really make a difference. You can work on engineering problems and be an engineer. That's okay. That's cool. You can get stuff done but I'd just be one more person against the tide who works against it and helps build up this solution to a big problem... I like how it was all about working as a team towards this larger, helpful goal."

Ethical Decision Making. Students often explained that, from a difficult ethical situation, they learned something about ethical decision making. Such learned outcomes could be divided into two categories: the impacts of ethical decisions and how one goes about making ethical decisions.

Students cited both the potential drawbacks to making an ethical or unethical decision as well as the positive benefits. When citing examples of negative impacts, interviewees described cases where they or someone they knew had lost friends or jobs because of taking an ethical step. They also mentioned the possibility of harming or endangering others or making more work for themselves if they failed to act ethically. There were also negative impacts to themselves if they were caught acting unethically, with one interviewee saying, "I learned just to take that first punishment. Although you may be scared or it may seem bad, if you lie about it or try to put it off, it's just going to become worse. At some point, you're going to get caught and it's going to be way worse than what it would have originally been." Occasionally, a student often cited positive benefits to acting ethically, such as how it made them feel or the potential to influence others.

Finally, students also cited insights they gained on how to make ethical decisions. These included learning to ignore certain people or viewpoints, listening to one's conscience, and doing the right thing even when you do not want to or when it might mean breaking the rules.

As an example, some interviewees discussed the influence others have in how such decisions are made. One student, Catherine, shared an observation about the role of popular or persuasive people in dictating the ethics of a group, having noticed, "a lot of people agreeing with someone simply because they voiced it better or they're able to persuade better or they were just louder.” 
She learned, "to accept that you will have to deviate from the group at times and it will suck sometimes but it's necessary for your own, moral survival/integrity.” Another student described strengthening his own ethics by resisting the urge to cheat, and that, by doing so, he was building the ability to resist the influence of others.

A few students learned about the importance of doing right, even when they did not feel like behaving that way. One student said that he learned from Boy Scouts the importance of, "Doing the right thing whenever and wherever you may be.” He later explained,

"I think that it's there's satisfaction to be had in doing the right thing. Even sometimes when it's hard or not the most desirable outcome. In the long run, the discomfort or whatever you may face isn't as bad as doing the wrong thing. The initial discomfort of doing the right thing in the end is always far less of a cost to pay I guess you could say.”

\section{Discussion and Conclusions}

A number of ideas can be connected across the three categories of analysis presented above. These include concern about the potential consequences and impacts of ethical decisions, the role of integrity and honesty in ethics, and the importance of other-oriented traits such as empathy.

One of the most notable common ideas was how interviewees discussed the consequences and impact of ethical decision-making. When defining ethics broadly, students would frequently cite the importance of doing right, despite the consequences. They also discussed the potential conflict that may arise when their own standards of ethical conduct conflict with the norms of society and others. Both of these themes reemerged in the analysis of outcomes that students learned from ethical situations. Some of the students managed to bring these ideas into their discussions of engineering ethics, most frequently when they talked about the impact of engineering decisions on stakeholders. As young engineers, it seems that some of the interviewees were attempting to incorporate their views of ethics, generally, with how they perceive their future work and careers. However, when making such connections, the students seemed to view themselves as more removed from engineering ethics situations than they did other general ethics situations.

Another thread that ran through each theme was the role of integrity and related traits, such as honesty. Students seemed to view such roles as important for both an ethical person and an ethical engineering to possess. When discussing what they learned from an ethical situation, students often said they learned the importance of such traits, or that they developed the traits after going through the experience.

Finally, another idea that was shared across all the analysis was being other-oriented, both in individual treatment of others and in considering the views and feelings of others through empathy. It is interesting that students very frequently described developing empathy when sharing what they learned from an ethical situation, yet it came up less (though was still present) when discussing ethics generally, and to an even lesser degree in the section about engineering ethics. This could be because the students do not yet explicitly see the role that empathy plays in engineering decisions. This is seen as a potential gap or opportunity in engineering education: 
students may arrive in university programs already synthesized to thinking about empathy, but the link to engineering ethics seems under explored. More research is needed to understand why the students so frequently describe other-oriented sentiments, especially empathy, when discussing ethics and their previous experiences in ethical situations, yet they do not make a very clear connection between such traits and their engineering work.

Looking at the findings even more generally reveals likely influences from all three of the major approaches to normative ethics. First, frequent references to following rules and norms in general - along with more specific comments about the importance of laws, rules, ethical codes, etc. suggest a deontological approach to ethics. Here the focus is on one's commitment to duties or rules. A second prevalent theme can be found in statements about traits or other individual characteristics that are positively associated with moral or ethical actors. Such remarks align with a virtue approach to ethics, where specific virtues or moral character receive primary emphasis. Third and finally, one can find evidence of consequentialism when students talk about the importance of considering the impacts or consequences of ethical decisions, although no students were found making explicit reference to utilitarianism or other consequentialist theories.

On one hand, the findings suggest that many students have commonsense understandings and perceptions that to some extent align with or reflect at least one of the predominant approaches to normative ethics. Further, a small but notable number of students made statements suggesting "post-conventional" understandings of ethics and morality, as in comments about the situated and contextual nature of morality and ethics. These findings could provide inspiration for developing ethics courses, workshops, and other interventions that are tuned to the pre-existing perspectives of students. On the other hand, it is notable that only one student seemed to demonstrate awareness for how ethics can be distinguished from morality, and few if any students made explicit reference to well-known ethical theories or frameworks, much less famous philosophers who were concerned with ethics and morality (e.g., Socrates, Kant, etc.). This suggests that ethics interventions in engineering should presume little in the way of relevant background knowledge among participating engineering students.

It is finally worth noting that the analysis and results presented here are both preliminary in nature and part of a much larger research project. An analysis of the full collection of 100+ interviews conducted with first-year engineering students will continue. This will allow the researchers to establish whether the range and frequency of themes identified in this paper correspond with the larger data set. This process will also involve looking at other themes which were outside the scope of this paper, including efforts to code specific experiences and influences (e.g., academic, extracurricular, religious, service/volunteer, etc.) that students mentioned as important for shaping their perceptions of ethics and morality, and analysis of student responses in other thematic areas (e.g., as related to ethical disengagement, perceptions of justice and fairness, ethical climate at their university, etc.). Follow-up interviews will be conducted with as many of these interviewees as possible during their fourth year of studies, allowing the research team to examine longitudinally how engineering student perceptions of ethics and social responsibility change during the course of their undergraduate education. 


\section{Acknowledgements}

This material is based upon work supported by the National Science Foundation under Grant No. 1449370. Any opinions, findings, and conclusions or recommendations expressed in this material are those of the author(s) and do not necessarily reflect the views of the National Science Foundation.

\section{References}

1 Fuentes, D. S., G. M. Warnick, B. K. Jesiek, and R. Davies (2016). A Longitudinal Study of Social and Ethical Responsibility Among Undergraduate Engineering Students: Preliminary Survey Results.

Proceedings of the American Society for Engineering Education (ASEE) Annual Conference and Exposition, New Orleans, LA, USA, June 26-29, 2016.

2 Barry, B. E., and J. R. Herkert. (2014). “Engineering Ethics.” In A. Johri and B. Olds (Eds.), Cambridge Handbook of Engineering Education Research (pp. 673-692). Cambridge and New York: Cambridge University Press.

3 Colby, A., and W. M. Sullivan. (2008). “Ethics Teaching in Undergraduate Engineering Education.” Journal of Engineering Education, 97(3): 327-338.

4 Carpenter, D. D., Harding, T. S., Finelli, C. J., Montgomery, S. M., \& Passow, H. J. (2006). Engineering students' perceptions of and attitudes towards cheating. Journal of Engineering Education, 95(3), 181-194.

5 Harding, T. S., Carpenter, D. D., Finelli, C. J., \& Passow, H. J. (2004). Does academic dishonesty relate to unethical behavior in professional practice? An exploratory study. Science and Engineering Ethics, 10 (2): 311-324.

6 Harding, T. S., Carpenter, D. D., \& Finelli, C. J. (2012). An exploratory investigation of the ethical behavior of engineering undergraduates. Journal of Engineering Education, 101(2), 346-374.

$7 \quad$ Holsapple, M., D. Carpenter, J. Sutkus, C. Finelli, and T. Harding. (2012). "Framing Faculty and Student Discrepancies in Engineering Ethics Education Delivery.” Journal of Engineering Education, 101(2): 169186.

8 Loui, Michael (2005). Ethics and the development of professional identities of engineering students. Journal of Engineering Education, 94(4), 383-390.

9 Feister, M. W. K., C. B. Zoltowski, P. M. Buzzanell, Q. Zhu, and W. C. Oakes. (2014a). "Making Sense of Ethics in Engineering Education: A Discursive Examination of Students' Perceptions of Work and Ethics on Multidisciplinary Project Teams.” Proceedings of the 2014 IEEE International Symposium on Ethics in Engineering, Science and Technology, Chicago, IL, May 23-24, 2014.

10 Feister, M. W. K., C. B. Zoltowski, P. M. Buzzanell, W. C. Oakes, and Q. Zhu. (2014). "Understanding Team Ethical Climate Through Interview Data.” Proceedings of the 2014 ASEE Annual Conference and Exposition, Indianapolis, IN, June 15-18, 2014.

11 Canney, N. E., \& Bielefeldt, A. R. (2012, June), A Model for the Development of Personal and Professional Social Responsibility for Engineers Paper presented at 2012 ASEE Annual Conference \& Exposition, San Antonio, Texas. https://peer.asee.org/20830

12 Bielefeldt, A. R., \& Canney, N. E. (2016, June), Perspectives of Engineers on Ethical Dilemmas in the Worksplace. Paper presented at 2012 ASEE Annual Conference \& Exposition, New Orleans, LA.

13 Rudnicka, E. (2009). Development and Evaluation of a Model to Assess Engineering Ethical Reasoning and Decision Making (Doctoral Dissertation). Pittsburg, PA: University of Pittsburg.

14 Feister, M. W. K., C. B. Zoltowski, P. M. Buzzanell, Q. Zhu, and W. C. Oakes. (2014a). "Making Sense of Ethics in Engineering Education: A Discursive Examination of Students' Perceptions of Work and Ethics on Multidisciplinary Project Teams.” Proceedings of the 2014 IEEE International Symposium on Ethics in Engineering, Science and Technology, Chicago, IL, May 23-24, 2014. 
15 Kenny Feister, M., Zoltowski, C. B.; Buzzanell, P. M., Torres, D. H. (2016, June), Integrating ethical considerations in design. Proceedings of 2016 ASEE Annual Conference Exposition, New Orleans, LA, June, 2016. doi:10.18260/p.25804

16 Sunderland, M. (2013). "Using Student Engagement to Relocate Ethics to the Core of the Engineering Curriculum.” Science and Engineering Ethics. doi: 10.1007/s11948-013-9444-5.

17 Eckles, R.E., Mesline, E.M., Gaffney, M., and Heift, P.R. (2005), Medical Ethics Education: Where Are We? Where Should We Be Going? A Review. Academic Medicine, 80(12) 1143-1152.

18 Smith, K.V. and Godfrey, N.S. (2002), Being a Good Nurse and Doing the Right Thing: A Qualitative Study. Nursing Ethics, 9(3): 301-312.

19 National Science Foundation (2016). National Science Board: science and engineering indicators 2016. Retrieved from https://nsf.gov/statistics/2016/nsb20161/\#/report/chapter-2/undergraduate-educationenrollment-and-degrees-in-the-united-states/undergraduate-enrollment-in-the-united-states on January 25, 2017. 